\title{
Combined Density Functional Theory and Microkinetics Study to Predict Optimum Operating Conditions of Si (100) Surface Carbonization by Acetylene for High Power Devices
}

Santhanamoorthi Nachimuthu, ${ }^{\mathrm{a}, \dagger}$ Tsung-Ruei Chen, ${ }^{\mathrm{a}, \dagger}$ Chen-Hao Yeh, ${ }^{\mathrm{b}}$ Lu-Sheng Hong, ${ }^{\mathrm{a}, *}$ and Jyh-Chiang Jiang, a,*

aDepartment of Chemical Engineering, National Taiwan University of Science and Technology, Taipei 106, Taiwan.

${ }^{\mathrm{b}}$ Department of Materials Science and Engineering, Feng Chia University, Taichung 40724, Taiwan

$\dagger$ These authors contributed equally to this work.

* Corresponding Authors e-mail

hongls@mail.ntust.edu.tw Telephone:+886-2-27376650. (LSH)

jcjiang@mail.ntust.edu.tw ; Telephone: +886-2-27376653. (JCJ) 


\section{Microkinetic Simulation}

In the microkinetic modeling, the rates of the adsorption $\left(r_{a d s}\right)$ and desorption $\left(r_{d e s}\right)$ of species A can be defined as:

$$
\begin{gathered}
r_{a d s}(t)=k_{a d s} \times Y_{A} \times \theta \\
r_{d e s}(t)=k_{\text {des }} \times \theta_{A}
\end{gathered}
$$

where $k$ is the rate constant, $\theta$ is the coverage on the surface, and $Y$ is the mole fraction in the gasphase. The principle of microscopic reversibility demands that a reaction passes through the same states irrespective of whether it proceeds forward or backward. The same set of rate equations can therefore describe adsorption and desorption. The desorption rate is usually expressed by a rate law of $n^{\text {th }}$ order:

$$
v=-\frac{\mathrm{d} \theta}{\mathrm{dt}}=k \times \theta^{n}
$$

, and the rate law is usually referred to as the Polanyi-Wigner equation:

$$
v=-\frac{\mathrm{d} \theta}{\mathrm{dt}}=k \times \theta^{n}=A(\theta, T) \exp \left(\frac{-E_{\text {des }}(\theta, T)}{k T}\right) \times \theta^{n}
$$

where $E_{\text {des }}(\theta, T)$ is the electronic energy for the desorption process, and $A(\theta, T)$ is the preexponential factor.

The general expression of a rate constant for an elementary reaction step can be written as follows,

$$
k=\frac{k_{B} T}{h} \times \frac{Q_{T S}^{\ddagger}}{Q_{I S}} \exp \left(\frac{-\mathrm{Ea}}{k_{B} T}\right)
$$

where $h$ and $k_{B}$ are the Plank and Boltzmann constants, respectively. $Q_{T S}^{\ddagger}$ and $Q_{I S}$ are the partition functions of the transition state and initial state, respectively. Ea is the activation energy between the reactant and the transition states. Also, we will extend this equation and apply it to solve the rate constants for adsorption, reactions with transition states, and desorption.

\section{Adsorption}

When a molecule adsorbs from the gas phase to the surface, any non-linear molecule possesses three translational degrees of freedom as well as three rotational degrees of freedom. All other degrees of freedom belongs to the internal vibrations. However, when the molecule is adsorbed on the surface, it loses the degrees of both translational and rotational freedom. Thus, after adsorption, the molecule has only vibrational degrees of freedom. According to the assumption of Hertz-Knudsen adsorption, for non-activated adsorption, the activation energy is zero. In addition, the transition state has two degrees of translational freedom, three rotational degrees of freedom, and $3 \mathrm{~N}-6$ vibrational degrees of freedom. Moreover, the initial state 
corresponds to the gas phase's molecule, including three rotational and three translational degrees of freedom. All other $3 \mathrm{~N}-6$ degrees of freedom are vibrational, as shown in the following equation.

$$
k=\frac{k_{b} T}{h} \times \frac{Q_{\text {trans }}^{\ddagger(2)} Q_{\text {rot }}^{\ddagger(3)} Q_{\text {vib }}^{\ddagger(3 N-6)}}{Q_{\text {trans }}^{(3)} Q_{\text {rot }}^{(3)} Q_{\text {vib }}^{(3 N-6)}}
$$

In the assumption of Hertz-Knudsen adsorption, the vibrational partition functions can be neglected as they approximate unity under typical reaction conditions, and there is no appreciable change in the rotational partition functions upon adsorption. Therefore, the equation can be rewritten as follows,

$$
k=\frac{k_{b} T}{h} \times \frac{L^{2}\left(2 \pi m k_{b} T\right) h^{3}}{L^{3}\left(2 \pi m k_{b} T\right)^{\frac{3}{2}} h^{3}}=\frac{k_{b} T}{L \sqrt{2 \pi m k_{b} T}}
$$

After assuming gas obeying the ideal gas law, we can obtain the formula as,

$$
k_{a d s}=\frac{P A}{\sqrt{2 \pi m k_{b} T}}
$$

where $\mathrm{P}$ denotes the partial pressure of the molecule in the gas phase, and A denotes the area of the surface site on which the molecule absorbs.

\section{Reactions with transition states}

Surface reactions involve the adsorbates proceed through a transition state. The energy difference between the initial and transition state is known as the activation energy or reaction barrier. As mentioned earlier, the surface species typically only have vibrational degrees of freedom. Therefore, the formula for the rate constant of a surface reaction is similar to eqn. (5), but the partition functions are only contributed by the vibrational and the reaction barrier $\left(E_{a}\right)$ between the reactant and the transition states.

$$
k=\frac{k_{B} T}{h} \times \frac{Q_{v i b}^{\ddagger(3 N-7)}}{Q_{v i b}^{(3 N-6)}} \exp \left(\frac{-E_{a}}{k_{B} T}\right)
$$

where $\mathrm{h}$ and $\mathrm{k}_{\mathrm{B}}$ are the Plank and Boltzmann constants, respectively. $Q_{v i b}^{\ddagger(3 N-7)}$ and $Q_{v i b}^{(3 N-6)}$ are the vibrational partition functions of the transition state and initial state in the surface reaction, respectively.

\section{Desorption}

Desorption is the opposite of adsorption: a bound compound leaves the catalytic surface and goes to the gas phase. In the previous section, we considered that in the transition state, the compound had three rotational degrees of freedom, two translational degrees of freedom, and 
$3 \mathrm{~N}-6$ vibrational degrees of freedom. Furthermore, we assume that in the initial state (the fully absorbed state), the compound only contains vibrational degrees of freedom, which results in the following expression for the reaction rate:

$$
\begin{gathered}
k=\frac{k_{b} T}{h} \times \frac{Q_{\text {trans }}^{\ddagger(2)} Q_{\text {rot }}^{\ddagger(3)} Q_{\text {vib }}^{\ddagger(3 N-6)}}{Q_{\text {vib }}^{(3 N)}} \exp \left(\frac{-E_{\text {des }}}{k_{b} T}\right) \\
=\frac{k_{b} T}{h} \times \frac{A\left(2 \pi m k_{b} T\right)}{h^{2}} \times \frac{8 \pi^{2} I k_{b} T}{\sigma h^{2}} \exp \left(\frac{-E_{\text {des }}}{k_{b} T}\right)
\end{gathered}
$$

where the $\sigma$ is the symmetry number and the $\frac{h^{2}}{8 \pi^{2} I k_{b}}$ is the rotational temperature $\theta_{\text {rot. }}$ Thus, the desorption rate constant is equal to:

$$
k=\frac{k_{b} T^{3}}{h^{3}} \times \frac{A\left(2 \pi m k_{b}\right)}{\sigma \theta_{\text {rot }}} \exp \left(\frac{-E_{\text {des }}}{k_{b} T}\right)
$$

The rate constants of each elementary reaction can be determined by the statistical thermodynamics combined with our DFT calculations. All microkinetic simulations were performed using MKMCXX software. ${ }^{1-2}$ By timely integrating the ordinary differential equations, the code could calculate the steady-state surface coverage and would terminate while the change in surface coverage was negligible. 

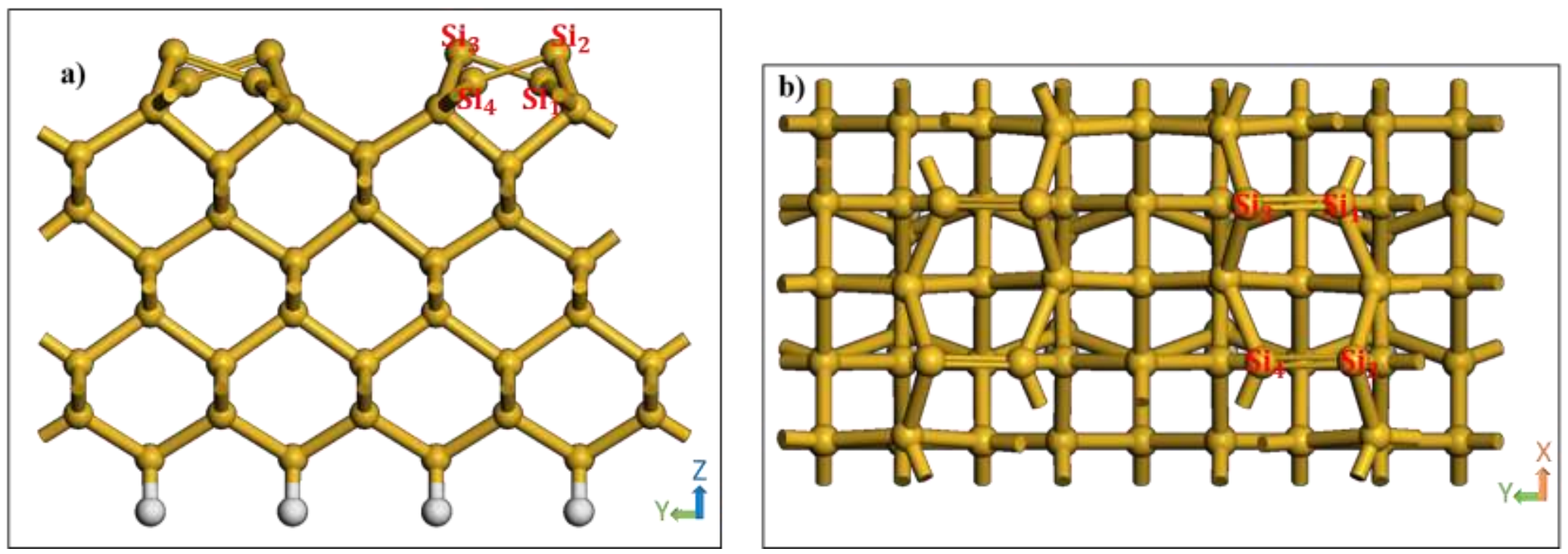

Figure 1S. a) Front and b) top views of the Si (100) surface model used in the present study. (Silicon Dimers are marked in the red) 


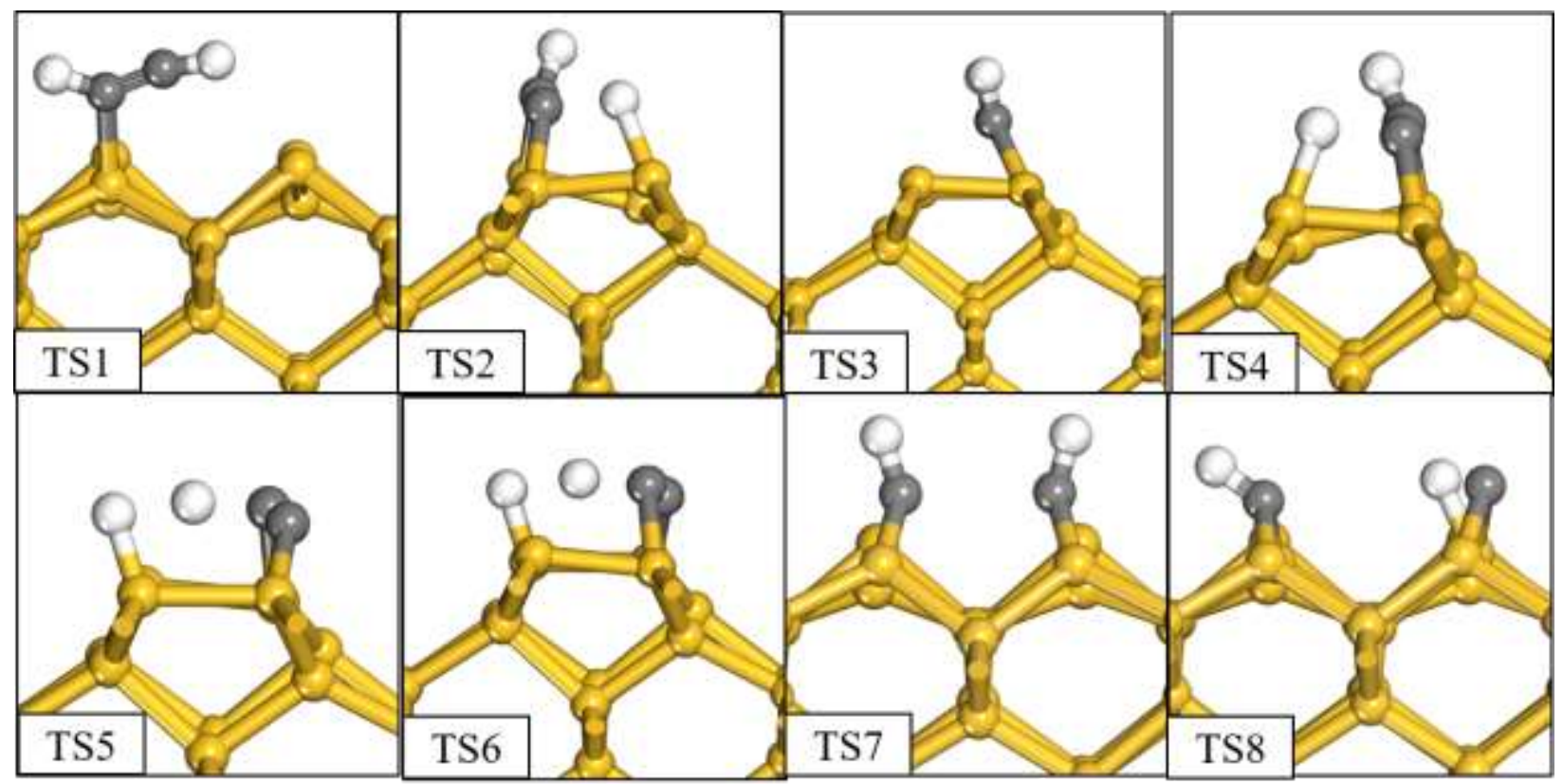

Figure 2S. Transition state structures for acetylene decomposition via end-bridge adsorption configuration (Color code: Silicon - Yellow, Carbon-Gray, Hydrogen-white) 


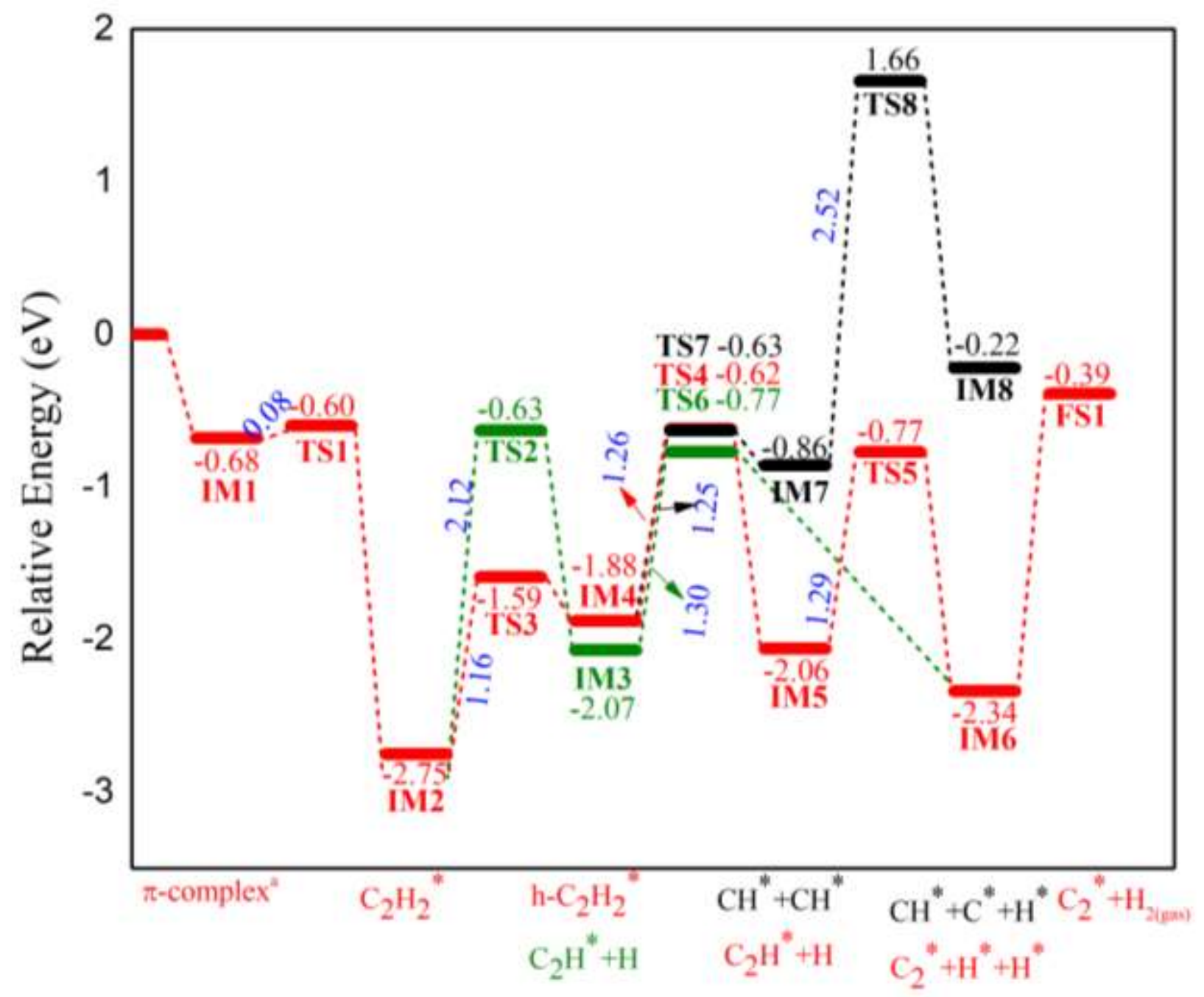

Figure 3S. The potential energy profile for the acetylene decomposition over Si (100) surface via end-bridge adsorption configuration. 


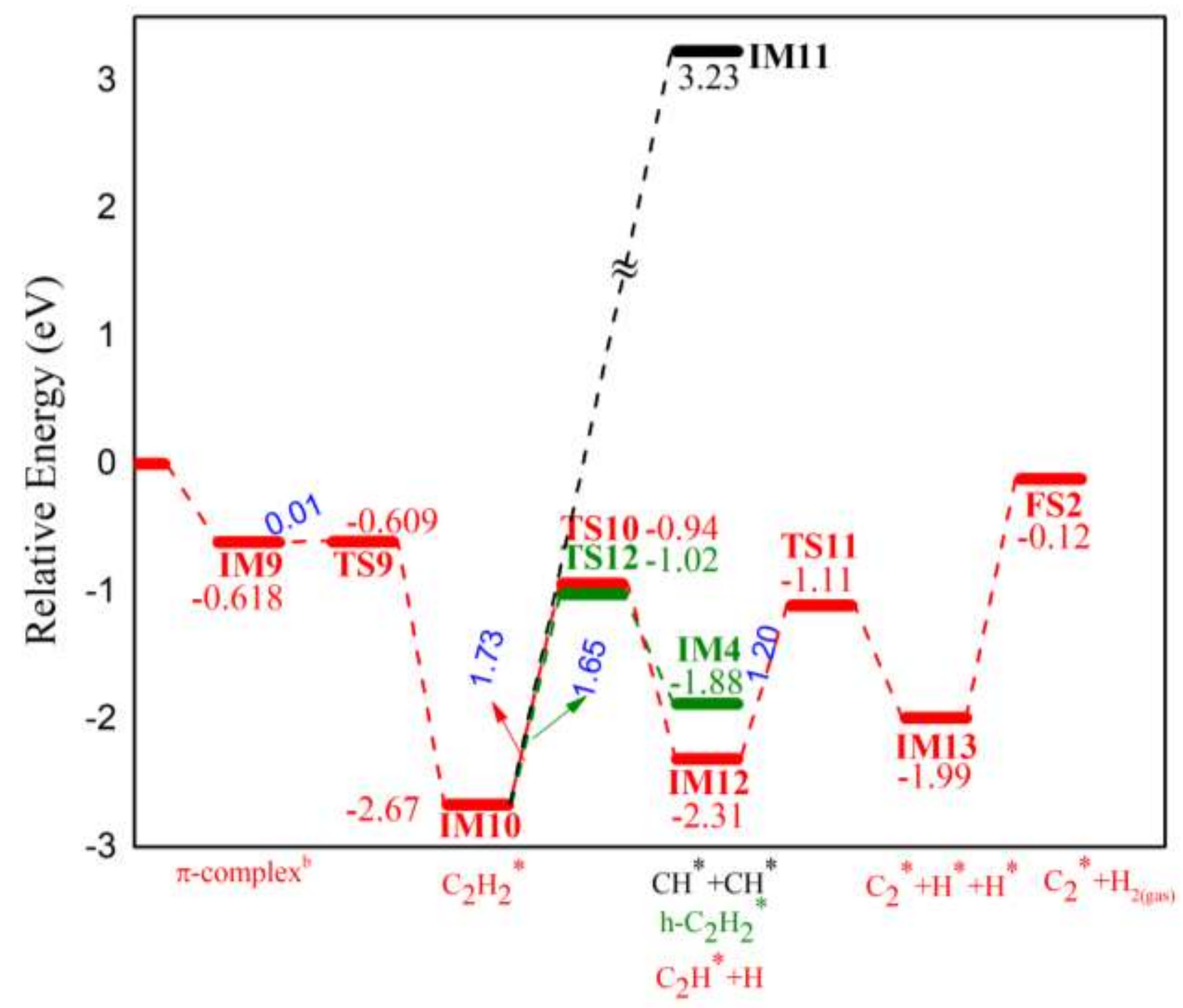

Figure 4S. The potential energy profile for the acetylene decomposition over Si (100) surface via di- $\sigma$ adsorption configuration. 


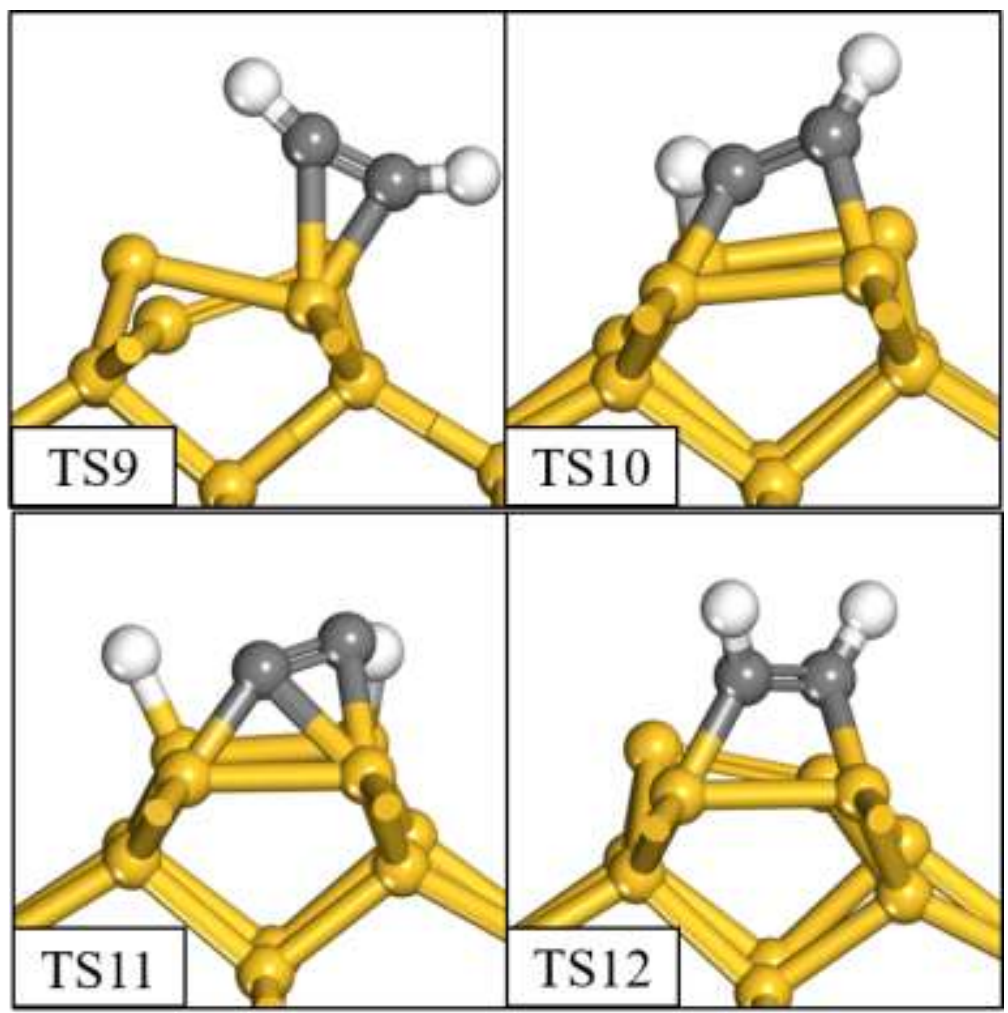

Figure 5S. Transition state structures for acetylene decomposition via di- $\sigma$ adsorption configuration (Color code: Silicon - Yellow, Carbon-Gray, Hydrogen-white) 


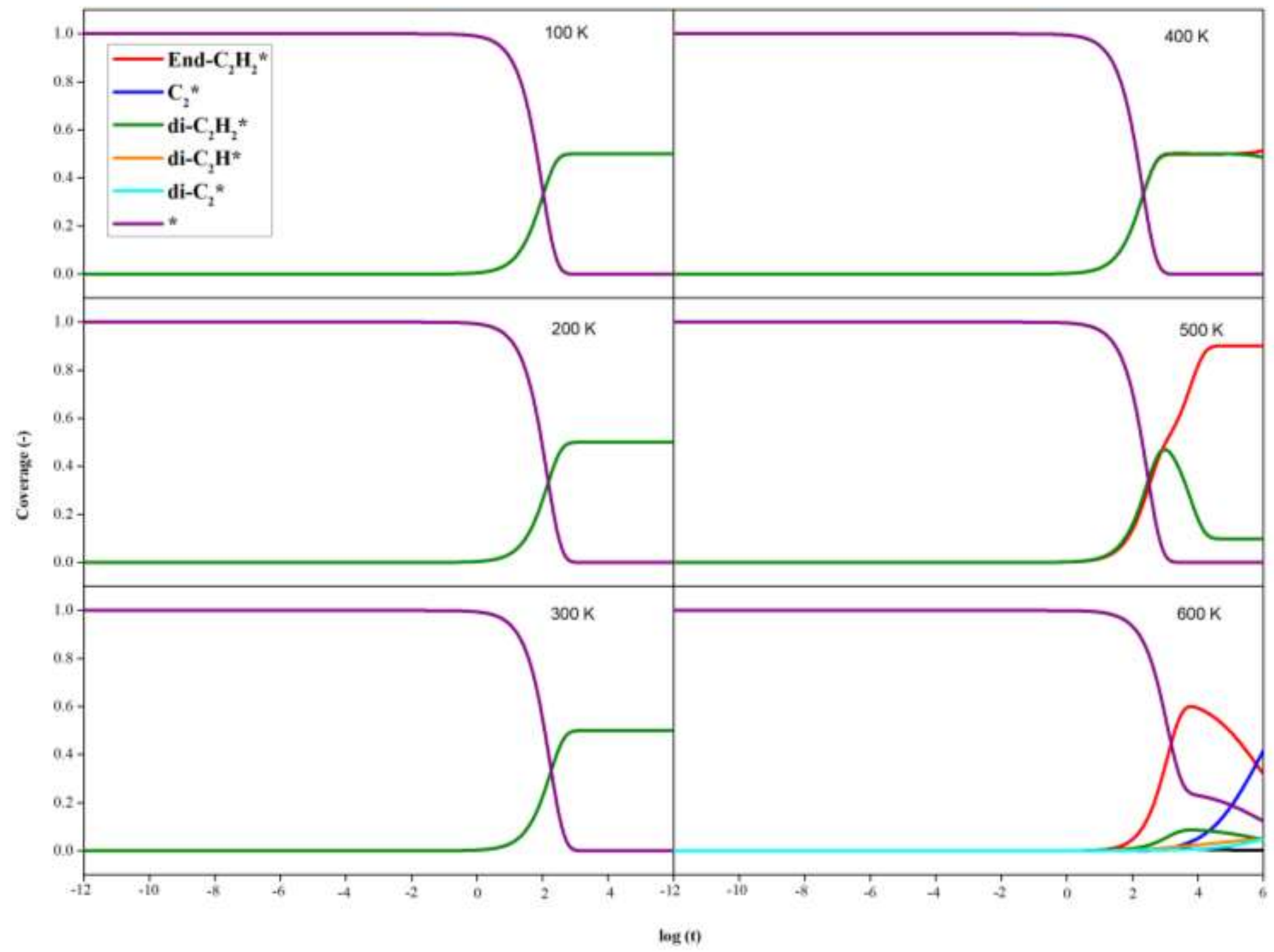

Figure 6S. The surface coverage of intermediates as a function of time upon Si carbonization by acetylene. 


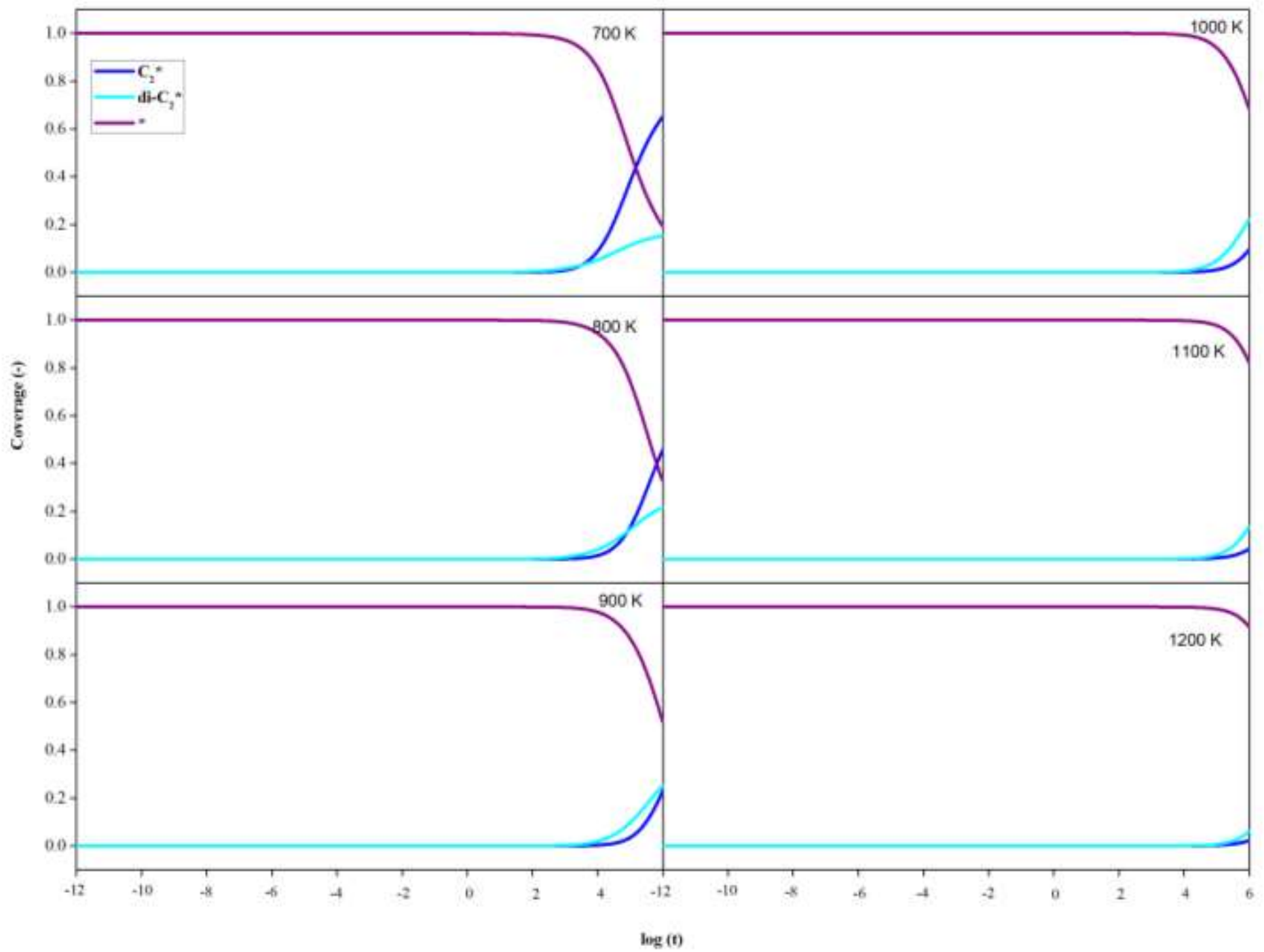

Figure 6S. (Continued) 


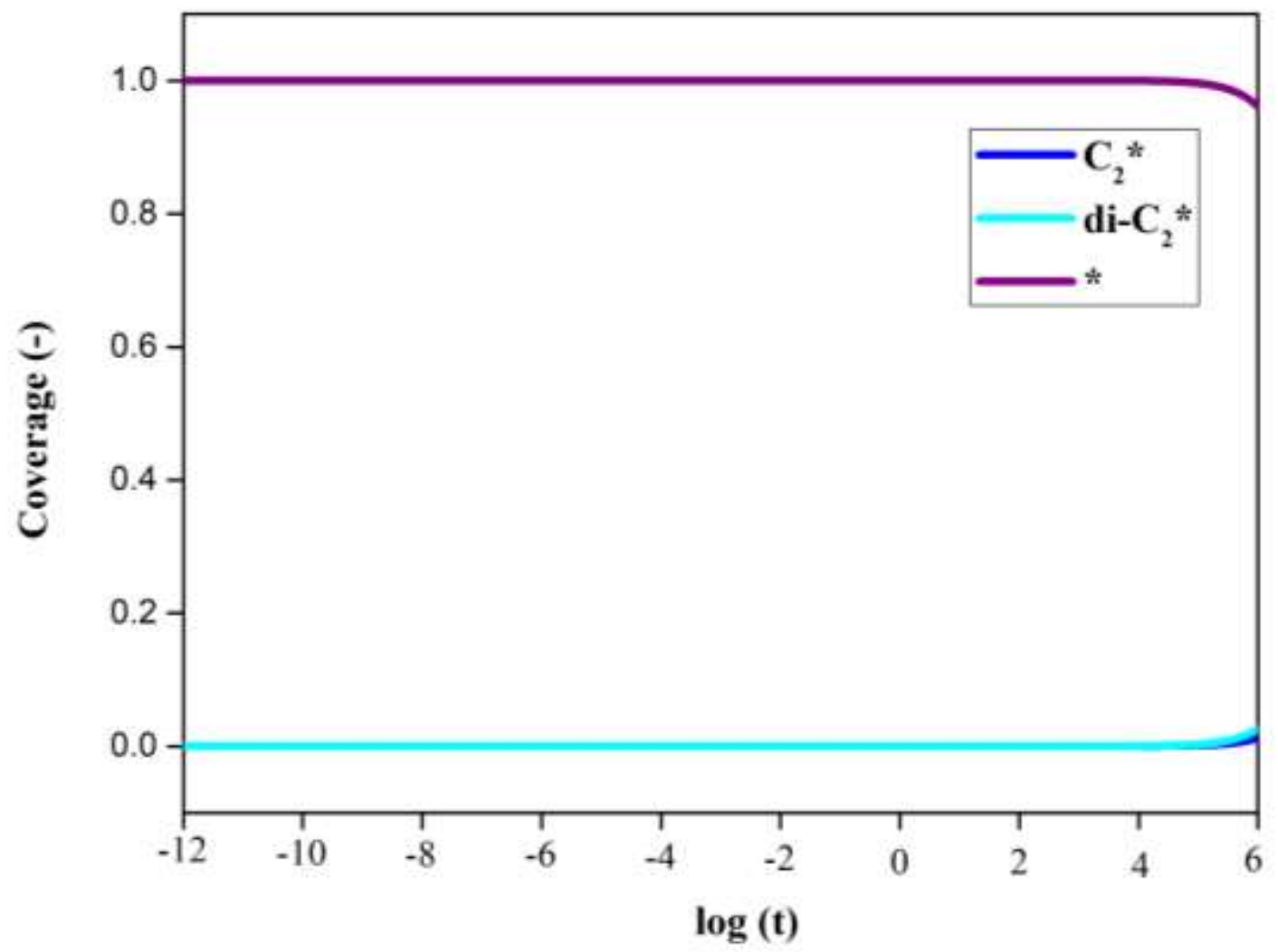

Figure 6S. (Continued)

\section{References}

1. Filot, I. A. W.; van Santen, R. A.; Hensen, E. J. M. The Optimally Performing Fischer-Tropsch Catalyst. Angew. Chem. Int. Ed. 2014, 53 (47), 12746-12750.

2. van Santen, R. A.; Markvoort, A. J.; Filot, I. A. W.; Ghouri, M. M.; Hensen, E. J. M. Mechanism and microkinetics of the Fischer-Tropsch reaction. Phys. Chem. Chem. Phys. 2013, 15 (40), 17038-17063. 